\title{
Diagnosis and Immunotherapy Strategies for Melanoma: A Review
}

\author{
Amrish Kumar*1, Dr. Vrish Dhwaj Ashwlayan", Mrs. Mansi Verma, Prof. (Dr.) Vipin Kumar Garg', Dr. Anurag \\ Chaudhary ${ }^{1}$, Mr. Avnesh Kumar ${ }^{1}$, Prof. (Dr.) Satish Kumar Gupta ${ }^{1}$, Dr. Anjana Sharma ${ }^{1}$, Dr. Sameksha Koul ${ }^{1}$, Dr. \\ Anoop Kumar ${ }^{1}$, Mrs. Garima Garg${ }^{1}$, Dr. Shobhit Kumar ${ }^{1}$, Mr. Firoj Khan ${ }^{2}$, Mr. Hement Tyagi ${ }^{3}$, Dr. Nitin Sharma ${ }^{4}$, Dr. \\ Lubhan Singh ${ }^{5}$ and Mr. Abhinav Agrawal ${ }^{6}$
}

${ }^{1}$ Department of Pharmaceutical Technology, Meerut Institute of Engineering and Technology, N.H. 58, Delhi-Roorkee Highway, Baghpat Bypass Road Crossing, Meerut, Uttar Pradesh 250005

${ }^{2}$ Clinical Research Coordinator, Jubilant Generics, Noida, Uttar Pradesh

${ }^{3}$ Department of Biotechnology, Meerut Institute of Engineering and Technology, N.H. 58, Delhi-Roorkee Highway, Baghpat Bypass Road Crossing, Meerut, Uttar Pradesh 250005

${ }^{4}$ Post Doc Fellow at Institute of Nuclear Medicine \& Allied Sciences (INMAS), Defence Research and Development Organisation, Brig. S.K. Mazumdar Marg, Delhi, 110054.

${ }^{5}$ Kharvel Subharti College of Pharmacy, Subhartipuram, NH-58, Delhi-Haridwar Bypass Road, Meerut, Uttar Pradesh.

${ }^{6}$ Raj Kumar Goel Institute of Technology, 5 KM STONE, Delhi-Meerut Rd, Ghaziabad, Uttar Pradesh 201003.

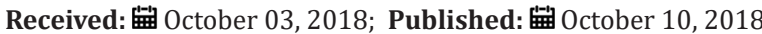

*Corresponding author: Amrish Kumar (Research Scholar, M.Pharm, Pharmacology), Department of Pharmaceutical Technology, Meerut institute of engineering and technology, Meerut, N.H. 58, Delhi-Roorkee Highway, Baghpat road crossing, Meerut, Uttar Pradesh 250005, India

\begin{abstract}
Melanoma is a type of skin cancer. Melanoma skin cancer is made up of abnormal pigment cells (melanocytes). Melanocytes are located at the bottom of the epidermis. These cells make melanin, which spreads to the top of the epidermis and gives skin its colour. Melanoma is usually brown or black in colour because the cell still makes melanin. Melanoma is more dangerous than other skin cancers because it more likely to spread if it is not found early. However, most melanomas about 84 out of 100 are found early before they have spread and so are likely to be cured with treatments. Melanoma can occur on any skin surface. In men, it's often found on the skin on the head, on the neck, or between the shoulders and the hips. In women, it's often found on the skin on the lower legs or between the shoulders and the hips. Melanoma is rare in people with dark skin. When it does develop in people with dark skin, it's usually found under the fingernails, under the toenails, on the palms of the hands, or on the soles of the feet. Although one of the less common types of skin cancer, melanoma is considered the most serious type of skin cancer because it is more likely to spread to other parts of the body, especially if not detected early. The earlier melanoma is found, the more successful treatment is likely to be. Despite the many investigations in this field and a rapidly growing knowledge base, classification according to specific mutational profiles is not yet validated. Further investigations are required for validation and refinement, and to possibly identify additional factors.
\end{abstract}

Keywords: Melanoma; Melanocytes; Epidermis; Melanin

\section{Introduction}

Cancer is a disease of the cells, which are the body's basic building blocks. The body constantly makes new cells to help us grow, replace worn-out tissue and heal injuries. Normally, cells multiply and die in an orderly way. Sometimes cells don't grow, divide and die in the usual way. This may cause blood or lymph fluid in the body to become abnormal, or form a lump called a tumour. A tumour can be benign or malignant.

\section{Benign tumour}

Cells are confined to one area and are not able to spread to other parts of the body. This is not cancer. 


\section{Malignant tumour}

This is made up of cancerous cells, which sometimes also have the ability to spread by travelling through the bloodstream or lymphatic system (lymph fluid) (Figure 1). The cancer that first develops in a tissue or organ is called the primary cancer. A malignant tumour is usually named after the organ or type of cell affected. A malignant tumour that has not spread to other parts of the body is called localized cancer. A tumour may invade deeper into surrounding tissue and can grow its own blood vessels in a process called angiogenesis. If cancerous cells grow and form another tumour at a new site, it is called a secondary cancer or metastasis. A metastasis keeps the name of the original cancer. For example, melanoma that has spread to the bones is called metastatic melanoma, even though the person may be experiencing symptoms caused by problems in the bones (Figure 2).
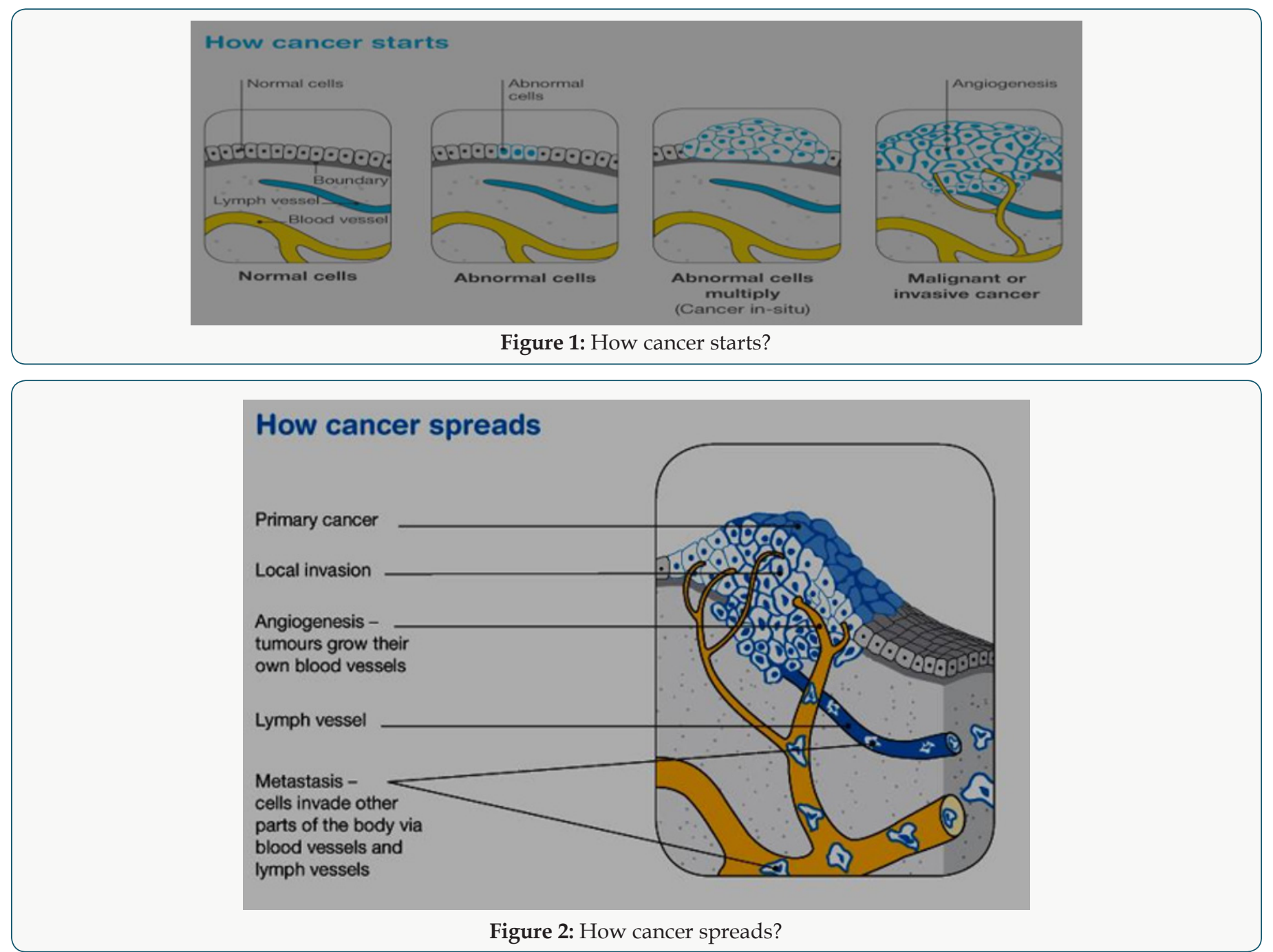

\section{The skin}

The skin is the largest organ in the body. It acts as a barrier to protect the body from injury, control body temperature and prevent loss of body fluids. Skin, like all other body tissues, is made up of cells. The two main layers of the skin are the epidermis and the dermis. Below these is a layer of fatty tissue (subcutis). The epidermis is the top, outer layer of the skin. It contains three main kinds of cells:

Squamous cells - These flat cells are packed tightly together to make up the top layer of skin and form the thickest layer of the epidermis. These cells eventually die and become the surface of your skin. Over time our body sheds these dead skin cells.

Basal cells: These block-like cells make up the lower layer of the epidermis and multiply constantly. As they age, they move upwards in the epidermis and flatten out to form squamous cells.

Melanocytes: These cells sit between the basal cells of the skin and produce a dark pigment called melanin, the substance that gives skin its colour. When skin is exposed to ultraviolet (UV) radiation, melanocytes make extra melanin to try to protect the skin from getting burnt. This is what causes skin to tan. Melanocytes are also 
in non-cancerous (benign) spots on the skin called moles or naevi. Most moles are brown, tan or pink in colour and round in shape [1].

\section{Melanoma}

All cancers involve the malfunction of genes that control cell growth and division. Most cancers are diagnosed in persons 55 and older. The lifetime risk for cancer is approximately $50 \%$ for men and approximately $33 \%$ for women. 1 About $5 \%$ to $10 \%$ of all cancers are clearly hereditary; the other $90-95 \%$ of cancers are not hereditary but result from damage to genes (that is, somatic mutations) due to internal factors (that is, hormones, digestion of nutrients) or external factors (such as tobacco, chemicals, and sunlight). Cancer treatment includes surgery, radiation, chemotherapy, and vaccines. Some cancers are treated using various combinations of surgery, radiation, and chemotherapy [2]. Basal cell carcinoma and squamous cell carcinoma are the most common skin cancers. The most serious common skin cancer is melanoma (malignant melanoma), which arises from neural crest-derived melanocytes located in the epidermis or dermis of the skin. Melanomas also can arise from melanocytes located in other regions of the body such as the eye, meninges, digestive tract, or lymph nodes. Melanocytes in the skin can give rise to a number of benign lesions such as nevi (moles), dysplastic nevi, Spitz nevi, blue nevi, solar lentigo, and seborrheic keratosis. When dealing with melanoma of the skin, establishing which pigmented lesions are malignant is the prime clinical problem, as the vast majority are benign.

\section{Epidemiology and Aetiology}

Exposure to the sun is the most commonly associated factor $[3,4]$. Predisposing factors that have been found to increase the risk of melanoma include skin that sunburns easily, poor tanning response, light-colored skin, history of severe sunburns, numerous nevi (more than 50), atypical nevi (that is, dysplastic nevi), a tendency toward freckling, a history of skin cancer, a family history of melanoma, the use of tanning salon, previous melanoma, and a weakened immune system (due to other cancers, transplant drugs, or HIV infection). Oral methoxsalen (psoralen) and ultraviolet A radiation is an effective treatment for psoriasis, but it is carcinogenic and increases the risk for melanoma. Individuals with recreational and vacation sun exposure may be at greater risk than those whose occupation exposes them to the sun constantly. In fact, continuous sun exposure appears to be a protective factor where melanoma is concerned.

Melanoma is a common cancer. In early 2005, the American Cancer Society estimated that approximately 59,500 individuals would be diagnosed with melanoma, the projected number of deaths was 7,770. The prevalence of melanoma is $13: 1,000$ Caucasian Americans. During the 1970s, the incidence rate of melanoma showed a marked increase of approximately $6.0 \%$ per year, although this rate slowed to approximately $3.0 \%$ a year beginning in 1981. There is some data to suggest that superficial spreading melanoma has been found more frequently during the last 30 years. Five-year survival rates for melanoma of the skin have increased from $80 \%$ in 1976 to $85 \%$ in 1985 to $91 \%$ in 2000 [5].

\section{Types of melanoma}

Superficial spreading melanoma: It begins with an intraepidermal horizontal or radial growth phase, appearing first as a macule that slowly evolves into a plaque, often with multiple colours and pale areas of regression. Secondary nodular areas may also develop. This is the most common type of melanoma, making up 55-60\% of all cases. It is more common in younger people and is often related to a pattern of irregular high sun exposure, including episodes of sunburn. It can start as a new brown or black spot that spreads within the outer layer of the skin (epidermis), or an existing spot, freckle or mole that changes size, colour or shape. It can develop on any part of the body. This type of melanoma often grows slowly and becomes more dangerous when it invades the lower layer of the skin (dermis) [6].

\section{Nodular melanoma}

Is a primarily nodular, exophytic brown-black, often eroded or bleeding tumour, which is characterized by an aggressive vertical phase, with a short or absent horizontal growth phase. This type makes up about $10-15 \%$ of melanomas. It usually appears as a round, raised lump on the surface of the skin that is often red, pink, brown or black and feels firm to touch. It may develop a crusty surface that bleeds easily. It is most commonly found in older people on severely sun-damaged skin on the head and neck. It is a fast growing and aggressive form of melanoma, spreading quickly into the lower layer of the skin (dermis) [7].

Lentigo maligna melanoma: Arises often after many years from a lentigo maligna (melanoma in situ) located predominantly on the sun-damaged faces of older individuals. This type of melanoma is most common in older people. It makes up about $10-15 \%$ of melanomas and begins as a large freckle (lentigo maligna) in an area of sun-damaged skin, such as the face, ears, neck and head. It may grow slowly and superficially over many years before it penetrates more deeply into the skin. Acral lentiginous melanoma: is typically palmoplantar or subungual. In its initial intraepidermal phase (which may be protracted), there is irregular, poorly circumscribed pigmentation; later a nodular region reflects the invasive growth pattern. This is an uncommon type of melanoma (around 1-2\% of all cases). It is most commonly found on the hairless skin on the soles of the feet or palms of the hands, or under the fingernails or toenails. It commonly appears as a colourless or lightly pigmented area, which can be mistaken for a stain or bruise. In the nails, it most often presents as a long streak of pigment in the nail or discoloration in the skin around the nail. It may grow slowly before it becomes invasive. 


\section{Desmoplastic melanoma}

This is another uncommon type of melanoma (around 1-2\% of cases) that presents as a firm, progressively growing lump, often on the head or neck. Many are skin-coloured and not pigmented and can be difficult to diagnose. There are some other rarer types of non-skin melanoma. Mucosal melanomas start in the tissues in the mouth, nervous system, anus, urethra, vagina, and nasal passages. Ocular melanomas start in the eye. Recent molecular studies have shown the genetic heterogeneity of melanoma, with distinct molecular signatures identified in tumours at different anatomical locations and with different associations with reported sun exposure.

\section{Symptoms of Melanoma}

Often the first sign of melanoma is a change in the shape, color, size, or feel of an existing mole. Melanoma may also appear as a new mole. Thinking of "ABCDE" can help you remember what to look for

a) Asymmetry: The shape of one half does not match the other half.

b) Border that is irregular: The edges are often ragged, notched, or blurred in outline. The pigment may spread into the surrounding skin.

c) Color that is uneven: Shades of black, brown, and tan may be present. Areas of white, gray, red, pink, or blue may also be seen

d) Diameter: There is a change in size, usually an increase. Melanomas can be tiny, but most are larger than the size of a pea (larger than 6 millimeters or about $1 / 4$ inch).

e) Evolving: The mole has changed over the past few weeks or months.

f) Risk and prevention: Exactly what cause of melanoma is unknown? But many risk factors for melanoma are known. A risk factor for melanoma is known. A risk factor is anything that increases the chance of getting a disease. Some risk factors are passed down from parent to child through genes. Other risk factors are activities that people do. Having one or more risk factors does not mean you will get melanoma. Likewise, melanoma occurs in some people who have no risk factors. Key melanoma risk factors are described next.

g) Ultraviolet energy: Melanoma often occurs on parts of the body exposed to UV energy. UV energy is an invisible light energy. The main source of UV energy or rays is sunlight. Tanning beds also expose the skin to UV rays and are known to cause skin cancer, including melanoma. Both UVA (ultraviolet A) and UVB (Ultraviolet B) rays contribute to the development of Melanoma and skin cancer. Too much exposure damages the skin and increases the risk for skin cancer. Whether sun exposure was too much depends on UV intensity, length of exposure, and how well the skin was protected [8].

\section{Diagnostic approach}

\section{Clinical and Dermoscopic diagnosis}

In most instances, the clinical appearance of melanoma varies according to the melanoma subtypes (see above). Typical features are asymmetry of the lesion, irregular borders, variability in colour, diameter of $5 \mathrm{~mm}$ and more, growth of nodules and regression of lesional components. The sensitivity of clinical diagnosis of experienced dermatologists is difficult to assess but estimated to be around 70\% [9]. The clinical diagnosis of the dermatologist is based on a combination of three analyses of any pigmented lesion: Visual analysis of each lesion separately which generally excludes non-melanocytic lesions, although melanomas may rarely mimic pigmented seborrhoeic keratoses. Examination with the naked eye assesses the so-called A (asymmetry), B (irregular borders), C (inhomogeneous colour) and D (diameter $5 \mathrm{~mm}$ ) criteria, which point to suspicious melanocytic lesions (ABCD rule). Intraindividual comparative analysis is searching for the nevus that is not alike the others in the same patient (ugly duckling sign) [10].

Chronologic analysis of change is looking for a rapid and recent change of a given pigmented lesion (E like evolution) at least when it can be attested by the patient or documented by comparison to previous pictures. Dermoscopy should be used to clarify the differential diagnosis of pigmented lesions. In order to apply this technique, training and expertise are required. A metaanalysis of 22 studies showed that when experts employed dermoscopy, they achieved an increase in diagnostic accuracy over the clinical diagnosis alone in questionable lesions and thus reached a sensitivity of $89 \%$ and a specificity of $79 \%$. Characteristic features for the diagnosis of melanoma, also called melanomaspecific criteria, include an atypical pigment network, irregular brown-black dots/ globules, streaks and pigmentation with multiple colours asymmetrically distributed. Additional criteria e.g. blue-whitish veil and polymorphic vessels are common in invasive melanoma. Amelanotic and featureless melanoma may represent a diagnostic challenge although suspicion should arise when a polymorphic vascular pattern is seen or when lesions do not display any of the well-known melanocytic or non-melanocytic characteristic dermoscopic features. This argues for urgent excision of any growing skin lesion suspicious for a skin tumour even if it looks more like a squamous lesion than a melanoma.

The prototypical dermoscopic progression model for LMM on the face include four sequential patterns, that are hyper-pigmented follicular openings, annulargranular pattern, rhomboidal structures and atypical pseudo-network, whilst the importance of additional features such as increased vascular network and red rhomboidal 
structures have been linked to the development of tumour-induced neovascularisation. Finally, a parallel ridge pattern and irregular diffuse pigmentation are distinguished features of early and invasive acral melanoma, respectively. In high risk patients, mainly in the case of patients with atypical mole syndrome, the detection of changes in the lesions or newly appearing lesions by follow-up examination with digital dermoscopy and total-body photography is also helpful. The differential diagnosis of melanoma involves other pigmented melanocytic lesions (congenital, atypical, common melanocytic naevi and actinic lentigo), non-melanocytic pigmented lesions (seborrhoeic keratosis, haemangioma, dermatofibroma, and pigmented basal cell carcinoma) and other non-pigmented tumours (haemangioma, basal cell carcinoma, squamous cell carcinoma). In addition to dermoscopy new non-invasive methods have recently been introduced in the clinical setting to increase accuracy in the diagnosis of equivocal lesions. Reflectance confocal microscopy increases specificity in equivocal dermoscopic melanocytic lesions in two prospective studies. This technology allows the diagnosis of subclinical lesions as amelanotic melanoma or better distinguishes the limits of the tumour [11] but has recently not been approved by NICE for routine use.

\section{Histopathologic diagnosis}

Whenever a suspicious skin lesion is removed a histological examination is warranted. Difficulties in the clinical diagnosis of melanoma can also be encountered on a histologic level. The specimen should be entrusted to a dermatopathologist experienced in the interpretation of pigmented lesions. The histopathologic report should include the following information [12]

a) Diagnosis and clinic-pathologic type, when there is uncertainty about malignancy it should be clearly stated in the report conclusion.

b) Tumour thickness in mm (Breslow's depth).

c) Presence or absence of ulceration.

d) Number of mitoses per mm2 (in hot spots).

e) Microsatellites (if present), defined as any discontinuous nest of intra-lymphatic metastatic cells of $>0.05 \mathrm{~mm}$ in diameter clearly separated by normal dermis or subcutaneous fat from the invasive component of the tumour by a distance of at least $0.3 \mathrm{~mm}$. f) Lateral and deep excision margins.

Besides these absolutely necessary histologic features, additional information can be provided, including:

a) A growth phase (horizontal or vertical).

b) A presence or absence of established regression.

c) A presence or absence of tumour infiltrating lymphocytes infiltrate preferably using the terms brisk, non-brisk or absent.

d) A Lymphatic embolus.

e) A Vascular or perineural involvement.

In some instances, when the histologic diagnosis is unclear, immunohistochemical stains may be helpful (i.e. S-100 protein, HMB45 and melan-A for the confirmation of the melanocytic nature of the tumour, HMB45 as an additional feature of malignancy when there is an inverted positive gradient, MIB-1 as a proliferation marker).

\section{Molecular diagnosis}

Molecular analysis of distant or regional metastasis or, if sampling of the metastatic tissue is not feasible, of the primary tumour is required for patients with distant metastasis or nonrespectable regional metastasis, who are candidates for systemic medical treatment [13]. Currently, the main test performed involves the BRAF V600 mutational status, in order to identify patients eligible for treatment with BRAF inhibitors and MEK inhibitors. NRAS mutations are identified in around 15\% of samples and as BRAF and NRAS mutations are mutually exclusive a positive NRAS mutation serves as to reassure that a BRAF mutation has not been missed. Presently, NRAS inhibitors are under clinical development [14]. NF1 mutations have been identified in around $10 \%$ of patients with CMs. Presently it is unknown, whether this type of mutation can be addressed with targeted therapies. CKIT mutations should additionally be analyzed in patients with acral and mucosal melanomas, although the positivity rate is lower than initially suggested in Europe. If present, patients can be treated with CKIT inhibitors. In the near future, other genomic tests are expected to be identified as predictive markers for patients with stage IV melanoma. In the future testing may even be performed in blood samples instead of tumour tissue ('liquid biopsy') based on extracellular circulating DNA or circulating tumour cells (Table 1).

Table 1: Staging of melanoma.

\begin{tabular}{|c|c|c|c|}
\hline Stage & Primary tumour (pT) & Regional lymph node metastases (N) & Distant metastases (M) \\
\hline 0 & In situ tumor & None & None \\
\hline IA & $\leq 1 \mathrm{~mm}$, no ulceration & None & None \\
\hline IB & $\leq 1 \mathrm{~mm}$ with ulceration & None & None \\
\hline
\end{tabular}




\begin{tabular}{|c|c|c|c|}
\hline IIA & $\begin{array}{l}1.01-2.00 \mathrm{~mm} \text {, with ulceration } \\
2.01-4 \mathrm{~mm} \text {, no ulceration }\end{array}$ & $\begin{array}{l}\text { None } \\
\text { None }\end{array}$ & $\begin{array}{l}\text { None } \\
\text { None }\end{array}$ \\
\hline IIB & $\begin{array}{l}\text { 2.01-4mm with ulceration } \\
>4 \mathrm{~mm} \text {, no ulceration }\end{array}$ & $\begin{array}{l}\text { None } \\
\text { None }\end{array}$ & $\begin{array}{l}\text { None } \\
\text { None }\end{array}$ \\
\hline IIIC & $>4 \mathrm{~mm}$, with ulceration & None & None \\
\hline IIIA & Any tumor thickness, no ulceration & Micrometastases & None \\
\hline IIIB & $\begin{array}{l}\text { Any tumor thickness with ulceration. } \\
\text { Any tumor thicknesses no ulceration. } \\
\text { Any tumor thickness } \pm \text { ulceration }\end{array}$ & $\begin{array}{l}\text { Micrometastases } \\
\text { Up to three macrometastases. } \\
\text { None but satellite and/or } \\
\text { in-transit metastases }\end{array}$ & $\begin{array}{l}\text { None } \\
\text { None } \\
\text { None }\end{array}$ \\
\hline IIIC & $\begin{array}{l}\text { Any tumour thickness with ulceration. } \\
\text { Any tumour thickness } \pm \text { ulceration }\end{array}$ & $\begin{array}{l}\text { Up to three macrometastases.Four or more } \\
\text { macrometastases, } \\
\text { or lymph node involvement } \\
\text { extending beyond capsule, or satellite and/ } \\
\text { or in-transit } \\
\text { metastases with lymph node involvement }\end{array}$ & $\begin{array}{l}\text { None } \\
\text { None }\end{array}$ \\
\hline IV & - & - & Distant metastases \\
\hline
\end{tabular}

\section{Immunotherapy}

Cytokines such as IFN-alpha and IL-2 were examined in several clinical trials in melanoma and achieved moderate response rates in non-controlled trials. Improvement of survival has never been shown in randomized clinical trials. Vaccination strategies have raised a lot of interest, but so far, no efficacious vaccines have been developed. In some trials, results may suggest even deleterious effects [15]. Blockade of the CTLA-4 and of the PD- 1 molecules expressed by lymphocytes abrogates down-regulation of immune responses and leads to continued activation of lymphocytes enabling killing of tumour cells. This immunostimulation is non-specific and can lead to immunologically mediated toxicity. The anti-CTLA-4 antibody ipilimumab was the first immunotherapy that showed a benefit for OS in two controlled trials in metastatic melanoma [1618]. Ipilimumab is approved for melanoma therapy in the USA and in the EU. It is presently administered as four intravenous infusions at a dose of $3 \mathrm{mg} / \mathrm{kg} /$ infusion separated by 3 weeks (Table 2).

Table 2: Dosage schedules for adjuvant therapy of melanoma with interferon-a.

\begin{tabular}{|c|c|c|c|c|}
\hline Schedule & Dose & Frequency & Duration & Indication \\
\hline Low dose & 3 million IU s.c. & Days 1,3 and 5 every week & Stage II- III \\
\hline High dose & 20 million IU $/ \mathrm{m}^{2}$ iv. & Day $1-5$ every week. & 4 weeks & Stage III \\
-Initiation & rapid infusion & Days 1,3 and 5 every week & 11 months & Stage III \\
-Maintenance & 10 million IU $/ \mathrm{m}^{2}$ s.c. & Day 1 every week & Stage III \\
\hline Pregylated & $6 \mathrm{mg} / \mathrm{kg}$ body weight s.c. & (up to 5 years) & Stage III \\
-Initiation & $3 \mathrm{mg} / \mathrm{kg}$ body weight s.c. & Day 1 every week & \\
-Maintenance & & &
\end{tabular}

Serious autoimmune reactions including skin rashes, colitis, thyroiditis, hepatitis, hypophysitis and others can develop in some patients and require interdisciplinary management. Early recognition of these reactions is mandatory and requires specific training of the caring physicians. The response rate to ipilimumab is only about $15 \%$, but remarkable durable remissions were observed in stage IV patients previously treated with other drugs. Patients with stable disease or initial disease progression may likewise 
benefit with prolonged survival. Meanwhile, the introduction of PD-1 antibodies changed the role of ipilimumab, which, in the future, will no longer be considered as the treatment of choice for first-line therapy, but ipilimumab will likely be used in combination with PD-1 antibodies (Table 3). The PD-1 antibodies nivolumab and pembrolizumab are approved for therapy of unresectable metastatic melanoma in the USA and Europe. Nivolumab was shown to improve progression-free and OS as compared to dacarbazine (CheckMate-066 trial) and as compared to ipilimumab (CheckMate-067 trial).

Table 3: Checkpoint blockade therapies for advanced cutaneous melanoma described in prospective randomised trials.

\begin{tabular}{|c|c|c|}
\hline Medication & Dose & $\begin{array}{l}\text { Response } \\
\text { rate }\end{array}$ \\
\hline Ipilimuma & $\begin{array}{c}3 \mathrm{mg} / \mathrm{kg} \text { i.v. every } 3 \text { weeks for four } \\
\text { cycles. }\end{array}$ & $12-19 \%$ \\
\hline Nivolumab & $\begin{array}{l}3 \mathrm{mg} / \mathrm{kg} \text { i.v. every } 2 \text { weeks } \\
\text { until tumour progression }\end{array}$ & $40-44 \%$ \\
\hline Pembrolizumab & $\begin{array}{c}2 \mathrm{mg} / \mathrm{kg} \text { i.v. every } 3 \text { weeks until tumour } \\
\text { progression }\end{array}$ & $33 \%$ \\
\hline Nivolumab & $\begin{array}{c}3 \mathrm{mg} / \mathrm{kg} \text { i.v. every } 3 \text { weeks for four } \\
\text { cycles. }\end{array}$ & \\
\hline $\begin{array}{l}\text { Nivolumab + } \\
\text { Ipilimumab }\end{array}$ & $\begin{array}{l}1 \mathrm{mg} / \mathrm{kg} \text { i.v. every } 3 \text { weeks for four } \\
\text { cycles, continuation with } 3 \mathrm{mg} / \mathrm{kg} \text { every } \\
2 \text { weeks until tumour progression }\end{array}$ & $58 \%$ \\
\hline
\end{tabular}

Pembrolizumab showed improved progression-free and OS in comparison to ipilimumab (KEYNOTE-006 trial). Objective response rates of w50\% were achieved with PD-1 blockade. However, longterm survival data are not yet widely available, but in some studies a 2 yearsurvival rate of $50 \%$ seems to be achieved by PD1- blokers. PD-1 blockade will be the first-line treatment of patients with BRAF wild-type tumours in future and may be considered for first-line treatment also in patients with BRAF mutation. The combination of nivolumab with ipilimumab was shown to be superior in progression-free survival to ipilimumab and to nivolumab as single drugs (Check- Mate-067 trial) leading to a FDA approval before OS data are available. However, there is clearly an increased toxicity as compared to the treatment with the single substances.

\section{Conclusion}

The frequency and extent of follow-up examinations depends on the primary tumour characteristics. The first 5 years following surgery are most important, as $90 \%$ of all metastases occur during this time period. Late metastasis does however occur in melanoma and indicate the relevance of a follow-up beyond 5 years. Patients who have had a history of melanoma have an increased risk of a secondary melanoma primary, adding increased importance to regular clinical reexaminations. Follow-up of melanoma patients has the following goals:

a) Identifying tumour recurrence or disease progression at the earliest stage. b) Diagnosing additional primary melanomas (occurs in about $10 \%$ of patients with $\mathrm{CM}$ ) at an early stage and nonmelanoma skin cancers.

c) Offering psychosocial support.

d) Providing education on prevention, for the patient and his first-degree relatives.

e) Providing education of the patient and his family on skin self-examination to promote the early detection of melanoma

\section{References}

1. (2017) Understanding Melanoma. Cancer Council Australia, ISBN: 978 1925136302.

2. (2005) Cancer Statistics for American Cancer Society.

3. Ferri FF (2005) Instant diagnosis and treatment. St. Louis: Mosby pp. 505-508.

4. Habif TP (2004) Clinical dermatology. A color guide to diagnosis and therapy, ed. 4. St. Louis: Mosby.

5. Cancer Statistics for American Cancer Society.

6. Habif TP (2004) Clinical dermatology. A color guide to diagnosis and therapy, ed. 4. St. Louis: Mosby.

7. O Hanlon KM, Plolak EF, Kabongo ML, Stevens SR, Rose L, et al. (2005) Melanoma. Elsevier.

8. (2018) NCCN guidelines for patients. Melanoma.

9. Gachon J, Beaulieu P, Sei JF, Gouvernet J, Claudel JP, et al. (2005) First prospective study of the recognitionprocess of melanoma in dermatological practice. Arch Dermatol 141(4): 434-438.

10. Grob JJ, Bonerandi JJ (1998) The ugly duckling sign: identification of the common characteristics of nevi in an individual as a basis for melanoma screening. Arch Dermatol 134(1): 103-104.

11. Alarcon I, Carrera C, Palou J, Alos L, Malvehy J, et al. (2014) Impact of in vivo reflectance confocal microscopy on the numberneeded to treat melanoma in doubtful lesions. Br J Dermatol 170(4): 802-808.

12. Ruiter DJ, Spatz A, Van den Oord JJ, Cook MG (2002) Pathologic staging of melanoma. Semin Oncol 29(4): 370-381.

13. Garbe C, Eigentler TK, Keilholz U, Hauschild A, Kirkwood JM (2011) Systematic review of medical treatment in melanoma. Current status and future prospects. Oncologist 16(1): 5-24.

14. Colombino M, Capone M, Lissia A, Cossu A, Rubino C, et al. (2012) BRAF/ NRAS mutation frequencies among primary tumors and metastases in patients with melanoma. J Clin Oncol 30(20): 2522-2529.

15. Morton DL, Mozzillo N, Thompson JF (2007) An international, randomized, phase III trial of bacillus Calmette-Guerin (BCG) plus allogeneic melanoma vaccine (MCV) or placebo after complete resection of melanoma metastatic to regional or distant sites. Edn 25(8): 8508.

16. Hodi FS, O Day SJ, McDermott DF, Weber RW, Sosman JA, et al. (2010) Improved survival with ipilimumab in patients with metastatic melanoma. N Engl J Med 363(8): 711-723.

17. Ko JM, Fisher DE (2011) A new era: melanoma genetics and therapeutics. J PATHOL 223(2): 241-250.

18. Sosman JA, Kim KB, Schuchter L, Gonzalez R, Pavlick AC, et al. (2012) Survival in BRAF V600-mutant advanced melanoma treated with vemurafenib. N Engl J Med 366(8): 707-714. 
(c) (P) This work is licensed under Creative

To Submit Your Article Click Here: $\quad$ Submit Article

DOI: $10.32474 /$ LOJMS.2018.02.000128

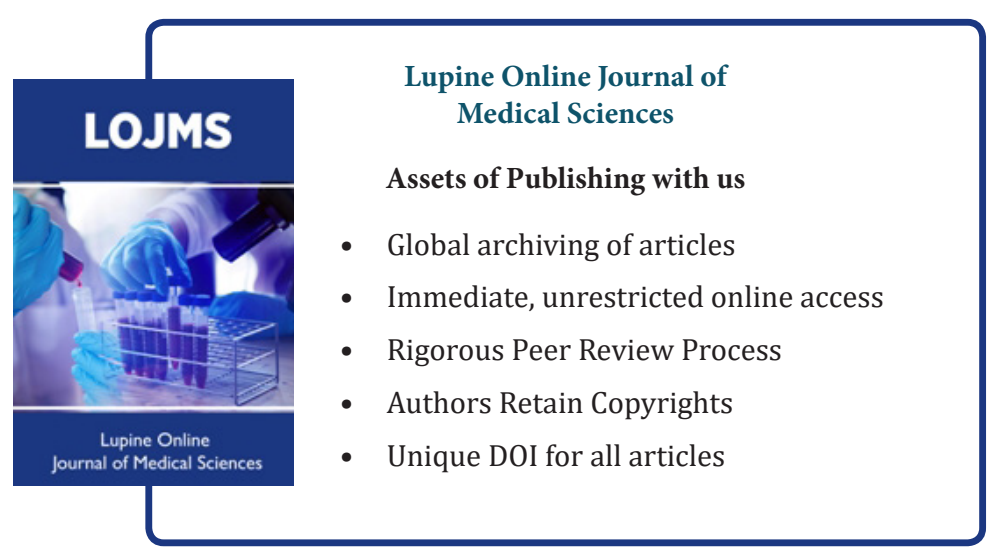

